GA-A23843

\title{
CONTROL OF NEOCLASSICAL TEARING MODES IN DIII-D
}

\author{
by \\ R.J. LA HAYE, S. GÜNTER, D.A. HUMPHREYS, J. LOHR, T.C. LUCE, \\ M.E. MARASCHEK, C.C. PETTY, R. PRATER, J.T. SCOVILLE, \\ and E.J. STRAIT
}




\section{DISCLAIMER}

This report was prepared as an account of work sponsored by an agency of the United States Government. Neither the United States Government nor any agency thereof, nor any of their employees, makes any warranty, express or implied, or assumes any legal liability or responsibility for the accuracy, completeness, or usefulness of any information, apparatus, product, or process disclosed, or represents that its use would not infringe privately owned rights. Reference herein to any specific commercial product, process, or service by trade name, trademark, manufacturer, or otherwise, does not necessarily constitute or imply its endorsement, recommendation, or favoring by the United States Government or any agency thereof. The views and opinions of authors expressed herein do not necessarily state or reflect those of the United States Government or any agency thereof. 
GA-A23843

\title{
CONTROL OF NEOCLASSICAL TEARING MODES IN DIII-D
}

\author{
by \\ R.J. LA HAYE, S. GÜNTER, * D.A. HUMPHREYS, J. LOHR, T.C. LUCE, \\ M.E. MARASCHEK, ${ }^{*}$ C.C. PETTY, R. PRATER, J.T. SCOVILLE, \\ and E.J. STRAIT
}

This is a preprint of an invited paper to be presented at the 43rd Annual Meeting of the Division of Plasma Physics in Long Beach, California, October 29 through November 2, 2001 and to be published in Phys. Plasma.

*Max-Planck Institut für Plasmaphysik, Garching, Germany.

\author{
Work supported by \\ the U.S. Department of Energy under \\ Contract No. DE-AC03-99ER54463
}




\section{ABSTRACT}

The development of techniques for neoclassical tearing mode (NTM) suppression or avoidance is crucial for successful high beta/high confinement tokamaks. Neoclassical tearing modes are islands destabilized and maintained by a helically perturbed bootstrap current and represent a significant limit to performance at higher poloidal beta. The confinement-degrading islands can be reduced or completely suppressed by precisely replacing the "missing" bootstrap current in the island O-point or by interfering with the fundamental helical harmonic of the pressure. Implementation of such techniques is being studied in the DIII-D tokamak [J.L. Luxon, et al., Plasma Phys. and Control. Fusion Research, Vol. 1 (International Atomic Energy Agency, Vienna, 1987) p. 159] in the presence of periodic $q=1$ sawtooth instabilities, a reactor relevant regime. Radially localized off-axis electron cyclotron current drive (ECCD) must be precisely located on the island. In DIII-D the plasma control system is put into a "search and suppress" mode to make either small rigid radial position shifts of the entire plasma (and thus the island) or small changes in toroidal field (and thus, ECCD location) to find and lock onto the optimum position for complete island suppression by ECCD. This is based on real-time measurements of an $m / n=3 / 2$ mode amplitude $d B_{\theta} / d t$. The experiment represents the first use of active feedback control to provide continuous, precise positioning. An alternative to ECCD makes use of the six toroidal section "C-Coil" on DIII-D to provide a large non-resonant static $m=1, n=3$ helical field to interfere with the fundamental harmonic of an $m / n=3 / 2$ NTM. While experiments show success in inhibiting the NTM if a large enough $n=3$ field is applied before the island onset, there is a considerable plasma rotation decrease due to $n=3$ "ripple". 


\section{INTRODUCTION}

Neoclassical tearing modes (NTMs) are islands destabilized and maintained by a helically perturbed bootstrap current and represent a significant limit to performance at higher poloidal beta in tokamaks. ${ }^{1}$ (Poloidal beta $\beta_{\theta}$ is the ratio of plasma pressure $p$ to poloidal magnetic field pressure $B_{\theta}^{2} / 2 \mu_{0}$.) The $m=3, n=2$ mode alone can decrease plasma energy by up to $30 \% .^{2}$ ( $m$ is the poloidal mode number and $n$ is the toroidal mode number for tearing modes resonant at safety factor $q=m / n$.) The $m=2, n=1$ node can have an even bigger deleterious effect and lead to disruption. ${ }^{3}$

The bootstrap current density $j_{\mathrm{bs}} \approx \varepsilon^{1 / 2} \cdot \nabla p / B_{\theta}$ arises at finite inverse aspect ratio $\varepsilon=r / R$ due to trapped particles and pressure gradient $\nabla p$ where $r$ and $R$ are the minor and major radii respectively. In the presence of a "seed" island of full width $w$, the flux surfaces are no longer nested as is shown in Fig. 1(a). A radial cut through the "O-point" is different in pressure than through the "X-point" as shown in Fig. 1(b). This assumes that parallel heat flow along field lines dominates over cross-field transport and flattens the pressure in the island, something to be addressed further in the paper. The zero $\nabla p$ at the O-point of the island produces a "missing" bootstrap current and together with the relatively unchanged bootstrap current at the X-point makes up a helically perturbed bootstrap current $\delta j_{\mathrm{bs}} \approx \varepsilon^{1 / 2} \cdot \delta \nabla p / B_{\theta}$ which is proportional to $\beta_{\theta} / w .{ }^{4,5}$ For conventional tokamaks with $q(r)$ increasing and $p(r)$ decreasing with minor radius, this $\delta j_{\mathrm{bs}}$ reinforces the seed island and is a destabilizing effect. Actual net stability also depends on other factors to be discussed but the nature of the NTM is a mode destabilized if $\beta_{\theta}$ is large enough.
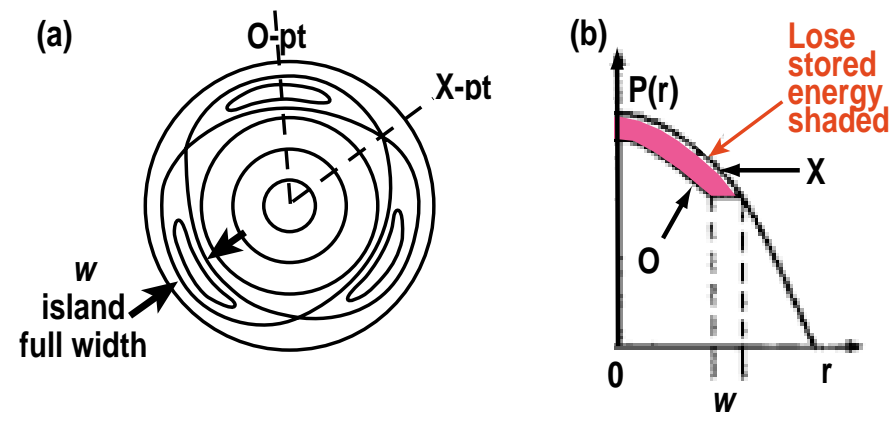

Fig. 1. (a) Torn flux surfaces of $m=3(n=2)$ islands of full width $w$. (b) Schematic of radial pressure profiles through $\mathrm{O}$-point and X-point with island $w$. 
Radially localized off-axis co-current drive could replace the "missing" bootstrap current in the island O-point and stabilize the NTM.6,7 This was confirmed both in ASDEX Upgrade, ${ }^{8}$ and in JT-60U ${ }^{9}$ with electron cyclotron current drive (ECCD). Periodic long-lived $q=1$ sawteeth instabilities can provide seed islands to trigger the $\mathrm{NTM}^{1}$ (and are expected to occur in reactor grade tokamaks such as ITER-EDA ${ }^{10}$ ). In ASDEX Upgrade, the sawteeth were often abated by the $m / n=3 / 2$ NTM and tended not to return with stabilization by the ECCD. The optimum location was found by a routine feed-forward sweep of toroidal field $B_{\mathrm{T}}$ so that the second harmonic resonance (and, thus, the ECCD) was on the island at some time during the sweep. In JT-60U, discharges were run with minimum $q>1$ to avoid sawteeth. The mirror launching the ECCD was swept to find the optimum angle for NTM suppression and then fixed at this position on subsequent discharges. In DIII-D, in the continued presence of $q=1$ sawteeth, ECCD is used to suppress the $3 / 2$ NTM by putting the plasma control system (PCS) into a "search and suppress" mode to make either small rigid radial position shifts of the entire plasma (and thus, the island) or small changes in toroidal field (and thus, ECCD resonance location) to find and lock onto the optimum position. This is based on real-time measurement of the mode amplitude $d B_{\theta} / d t$ and constitutes the first use of active feedback control to provide continuous, precise positioning.

An alternative to replacing the "missing" bootstrap current in the O-pt is to inhibit it. Partial flattening of the pressure in the island occurs if the parallel transport along field lines $\chi_{\|}$does not dominate over transport across field lines $\chi_{\perp} \cdot{ }^{11}$ A large increase in $\chi_{\perp}$ would "wash out" the perturbed bootstrap current but is undesirable in itself. Experiments on ASDEX Upgrade found that the existence of more than one NTM at a time usually did not occur. This was explained as the helical field of one mode $(m / n=3 / 2$ for example) interfering with the fundamental harmonic of another $(m / n=4 / 3$ for example) so as to weaken the helically perturbed bootstrap current enough to produce stabilization. ${ }^{12}$ It was then suggested that application of a large, preferably non-resonant, helical field from an external coil could do the same. ${ }^{13}$ This has the attractive features that the applied helical field could inhibit multiple NTMs $(m / n=4 / 3,3 / 2,2 / 1$, etc.) at the same time and does not require precise location. In DIII-D, use is made of the six toroidal section "C-Coil" (usually used to correct $n=1$ error fields) to apply a large non-resonant static $m=1$, $n=3$ helical field to interfere with the fundamental harmonic of an $m / n=3 / 2$ NTM. This is the first experimental use of large externally applied non-resonant fields to control a neoclassical tearing mode.

In Section 2, the control of a 3/2 NTM by ECCD is described including the real-time feedback to find and lock onto the optimum position for complete stabilization. Section 3 relates the effect of the $n=3$ field on a $3 / 2$ NTM and how applying the $n=3$ field before the high $\beta_{\theta}$ can inhibit the growth of the $3 / 2$ NTM until such time as the $n=3$ field itself is turned off. Conclusions are given in Section 4. 


\section{ECCD SEARCH AND SUPPRESSION OF AN NTM}

\section{A. MODIFIED RUTHERFORD EQUATION WITH RADIALLY LOCALIZED OFF-AXIS CURRENT DRIVE}

The NTM is metastable in that the high $\beta_{\theta}$ plasma without the island must be excited above a threshold island width for the island to grow large and saturate. This is shown by the modified Rutherford equation and in Fig. $2 .{ }^{1} \mathrm{~A}$ term for the ECCD is included. 6,7

$$
\frac{\tau_{\mathrm{R}}}{r} \frac{d w}{d t}=\Delta^{\prime} r+\varepsilon^{1 / 2}\left(\frac{L_{\mathrm{q}}}{L_{\mathrm{p}}}\right) \beta_{\theta}\left[\frac{r w}{w^{2}+w_{\mathrm{d}}^{2}}-\frac{r w_{\mathrm{pol}}^{2}}{w^{3}}-\frac{8 q r \delta_{\mathrm{ec}}}{\pi^{2} w^{2}}\left(\frac{\eta j_{\mathrm{ec}}}{j_{\mathrm{bs}}}\right)\right],
$$

and

$$
\eta=\eta_{0}\left(1+2 \delta_{\mathrm{ec}}^{2} / w^{2}\right)^{-1} \exp \left[-\left(5 \Delta R / 3 \delta_{\mathrm{ec}}\right)^{2}\right]
$$

with $j_{\mathrm{ec}} / j_{\mathrm{bs}}$ the ratio of the peak co-ECCD current density normalized to the local equilibrium bootstrap current density. The radio frequency (rf) efficiency $\eta$ has a coefficient $\eta_{0} \equiv 0.4$ for no modulation and allows for a reduction if the peak ECCD is not placed precisely $(\Delta R=0)$ on the island O-pt and/or if the ECCD width is greater than that of the island $\left[\delta_{\mathrm{ec}}\right.$ is the full radial width-half maximum (FWHM) of a Gaussian rf current density]. All other terms are defined in Ref. [1]. For no rf, the island would be excited above a threshold width and grow to a large saturated value for typical DIII-D parameters given in Fig. 2. The minor radius $r$ is taken at the $q=3 / 2$ surface on the midplane with respect to the effective major radius of the separatrix surface $R_{\text {surf }}$. By applying precisely located $j_{\mathrm{ec}}$ of $\geq 1.5 j_{\mathrm{bs}}$ it is predicted that the island can be reduced to a level such that complete suppression should occur. Any less $j_{\mathrm{ec}} / j_{\mathrm{bs}}$ or reduced rf efficiency $\left(\Delta R / \delta_{\mathrm{ec}} \neq 0\right)$ should lead to only a partial suppression. For FWHM $\delta_{\mathrm{ec}}=3 \mathrm{~cm}$, a misalignment of only $1.9 \mathrm{~cm}$ reduces the predicted rf efficiency by a factor of 3 . Integrating radially over the assumed Gaussian rf current density gives the total rf current $I_{\mathrm{ec}}$. This is about $2 \%$ of the plasma current $I_{\mathrm{p}}$ within $q=3 / 2$ for the $j_{\mathrm{ec}} / j_{\mathrm{bs}}=1.5$ case.

Coupling to other instabilities such as the $q=1 \mathrm{~m} / n=1 / 1$ and $2 / 2$ sawtooth precursors which can act to drive "seed" islands is not included in Eq. (1). Such coupling can both make the destabilizing seed ( $w \geq 2 \mathrm{~cm}$ in Fig. 2) and inhibit the suppression by rf, to be discussed later. 


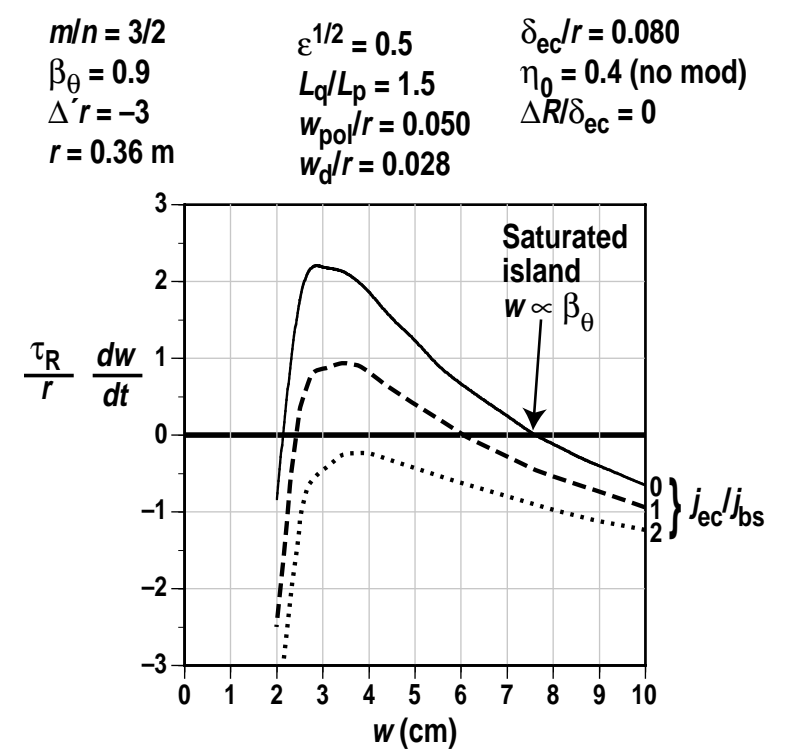

Fig. 2. Island growth (decay) rate calculated for different levels of precisely aligned peak rf current density.

\section{B. CONFIGURATION FOR OFF-AXIS ECCD IN DIII-D}

ELMing H-mode discharges with sawteeth were run in DIII-D in which large $m / n=3 / 2$ NTMs were made and allowed to saturate $(w / r \approx 20 \%)$. The periodic sawteeth continued in the presence of the NTM island. A cryopump was used to reduce the electron density (and concomitantly increase the electron temperature) so as to improve the rf current density driven $\left(j_{\mathrm{ec}} \propto P_{\mathrm{ec}} T_{\mathrm{e}} / n_{\mathrm{e}}\right.$ is expected). ${ }^{14,15}$ The injected rf power $P_{\mathrm{ec}}$ is up to $2.3 \mathrm{MW}$ for 2 seconds from four gyrotrons. The second harmonic resonance $2 f_{\mathrm{ce}}$ for the $110 \mathrm{GHz}$ gyrotron frequency is placed on the inboard midplane near the $q=3 / 2$ location as shown in Fig. 3. This tends to improve $j_{\mathrm{ec}}$ over an outboard resonance location where electron trapping effects are larger. 16 Two separate launchers are used, each with two gyrotrons; the launchers are independently steerable between discharges but not during a discharge. The rf absorption at the third harmonic resonance is expected to be small. The launchers are configured to maximize off-axis $j_{\mathrm{ec}}$ rather than to maximize the total driven current $I_{\mathrm{ec}}$.

Discharges are run with 2, 3, or 4 gyrotrons after $m / n=3 / 2$ mode saturation. Plasma current $I_{\mathrm{p}}$ is $1.0-1.2 \mathrm{MA}$, toroidal field $B_{\mathrm{T}}=-1.52$ to $-1.64 \mathrm{~T}$, line-averaged density $\bar{n} \approx 4 \times 10^{19} \mathrm{~m}^{-3}$, local $q=3 / 2 T_{\mathrm{e}} \approx T_{\mathrm{i}} \approx 1200-1600 \mathrm{eV}$, with safety factor at the $95 \%$ flux surface $q_{95}=3.2-4.3$. 


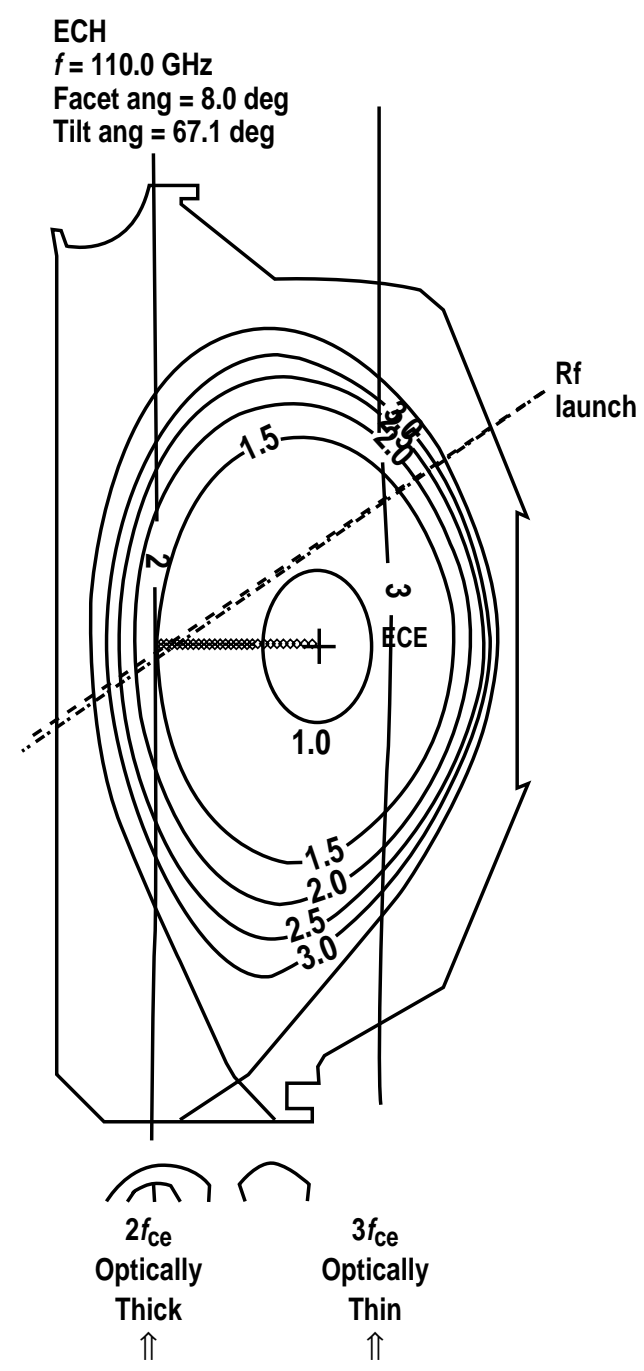

Fig. 3. Configuration for ECCD/NTM suppression showing $q=3 / 2$ surface, $2 f_{\text {ce }}$ location and projection of rf trajectory to crossing near $2 f_{\text {ce }}$ and $q=3 / 2$ on inboard midplane.

\section{DYNAMIC AND SHOT-TO-SHOT TOROIDAL FIELD ADJUSTMENT FOR FINDING THE OPTIMUM ECCD SUPPRESSION OF NTM}

The location of the ECCD is critical for optimum NTM stabilization. The location of the peak $j_{\mathrm{ec}}$ is a little outboard of the $2 f_{\mathrm{ce}}$ radius due to Doppler shift from finite $T_{\mathrm{e}}$. Topologically, $j_{\text {ec }}$ can be aligned on a $q=3 / 2$ island by adjusting the mirror angle, modifying $B_{\mathrm{T}}$ or moving the plasma (of major radius $R_{\text {surf }}$ ) rigidly to adjust the major radius of $q=3 / 2$.

The ASDEX Upgrade work ${ }^{8}$ relies on a feed forward slow sweep of the toroidal field in each discharge and, thus, the second harmonic resonance, so that at some time during 
the sweep the positioning is transiently correct. In JT- $60 \mathrm{U}^{9}$ the optimum rf wave injection angle was determined by scanning a steerable mirror during a discharge and then fixed in a subsequent discharge at the optimum angle (as determined from the dip in Mirnov amplitude during the scan). DIII-D uses a $B_{\mathrm{T}}$ sweep to find the optimum and then a fixed $B_{\mathrm{T}}$ at the optimum in subsequent discharges.

In DIII-D, the sawteeth continue even in the presence of the $m / n=3 / 2 \mathrm{NTM}$ and cause the on-axis $q$ to vary from $0.85-1.00$ which can also affect the $q=3 / 2$ location. Thus, time-to-time and shot-to-shot variations are both of concern for precise ECCD location. The optimum is first found by a $B_{\mathrm{T}}$ sweep as shown in Fig. 4, too fast to achieve complete suppression with only two gyrotrons. The TORAY-GA prediction ${ }^{17}$ of $j(\rho)$, where $\rho$ is the normalized minor radius, is shown for $1 \mathrm{MW}$ for the three times indicated along with the location and width of the initial island [from fast ECE radiometer $\tilde{T}_{\mathrm{e}}(R)$ at the $n=2$ Mirnov frequency]. These profiles show: (1) the need for alignment within $\pm 1 \mathrm{~cm}$ and (2) $j_{\mathrm{ec}} / j_{\mathrm{bs}} \approx 1.5$ is marginal in agreement with Fig. 2 . The $j_{\mathrm{bs}}$ comes from analysis by code ONETWO. ${ }^{18}$ Here the $n=2$ Mirnov rms amplitude comes from Fourier analysis of a toroidal array of Mirnov probes after integrating $d B_{\theta} / d t$.

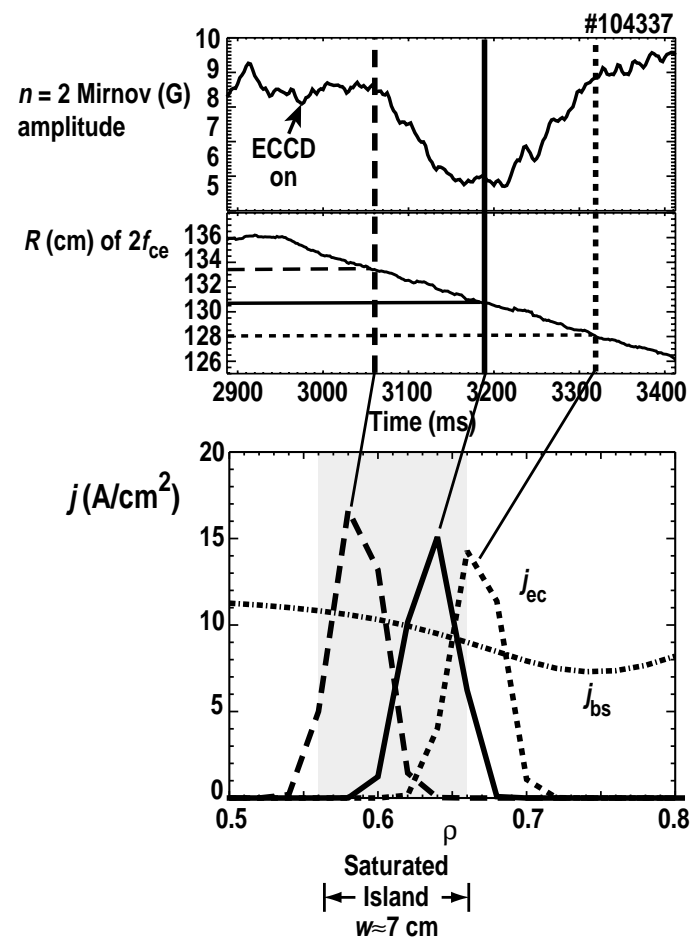

Fig. 4. (a) $B_{\mathrm{T}}$ sweep to move $2 f_{\text {ce }}$ location past island. ECCD on at $3000 \mathrm{~ms}$ ( 2 gyrotrons for $\approx 1 \mathrm{MW}$ injected) (b) calculated $j_{\mathrm{ec}}$ from TORAY-GA [at the three different times indicated in (a)] and $j_{\mathrm{bs}}$ from ONETWO code reconstruction [at the middle time indicated in (a)]. Island width and location from ECE radiometer at first time indicated in (a). 
Best results (i.e. complete $3 / 2$ NTM suppression) occur by setting the flattop $B_{\mathrm{T}}$ to the value of the biggest $n=2$ Mirnov dip of Fig. $4 . \beta_{\mathrm{N}}$ increases by about $25 \%$ as the $3 / 2$ NTM is suppressed and remains at this level even after the ECCD is turned off, as shown in Fig. 5. This case \#104328 has $q_{95}=3.2$, and periodic $q=1$ sawteeth are uncoupled from $q=3 / 2$, i.e., the $n=2$ Mirnov frequency $f_{32}$ is substantially higher than twice the $n=1$ Mirnov frequency $f_{11}$. The initial $j_{\mathrm{ec}} / j_{\mathrm{bs}} \approx 1.8$.

The importance of fine tuning $B_{\mathrm{T}}$ to place the $2 f_{\text {ce }}$ location (and thus ECCD) on the $3 / 2$ island is further shown in Fig. 6. Before the ECCD is applied, the $n=2$ Mirnov amplitude $\left|\tilde{B}_{\theta, 32}\right|$ is in steady state, i.e., $d w / d t=0$ and all terms on the right-hand side (RHS) of Eq. (1) add to zero. Upon turning on the rf, jnitially the RHS has only the rf term. Keeping all quantities fixed, initial $w \propto\left(\left|\tilde{B}_{\theta, 32}\right|\right)^{1 / 2}, n_{\mathrm{e}}, T_{\mathrm{e}}, P_{\mathrm{rf}}$, etc., the initial decay rate $\gamma$ is $\propto \exp \left[-\left(5 \Delta R / 3 \delta_{\mathrm{ec}}\right)^{2}\right]$ where $\Delta R$ is the misalignment. A shot-to-shot scan is shown in Fig. 6 in flattop $B_{\mathrm{T}}$ for the $q_{95}=3.2$ case with sawteeth "uncoupled" to the $3 / 2$ island, i.e. $2 f_{11} \neq f_{32}$. The FWHM width $\delta_{\text {ec }}$ is somewhat wider than predicted by TORAY-GA. This may be due to the model, difference in the current drive locations of the two gyrotrons or radial diffusion (not included in TORAY-GA) broadening the effective current drive width. ${ }^{19}$ The fit shows that a factor of 2 reduction in effectiveness occurs for a misalignment of only $1.9 \mathrm{~cm}$.

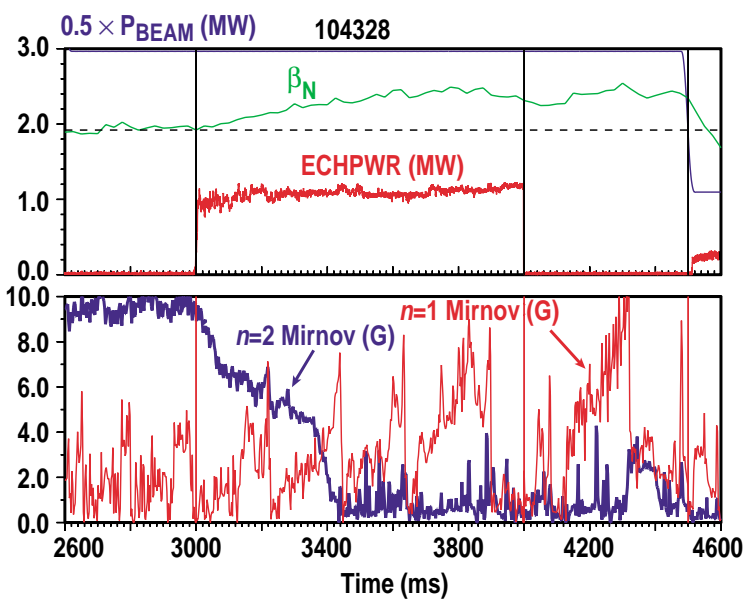

Fig. 5. Complete suppression of an $m / n=3 / 2$ NTM by ECCD in the presence of continued uncoupled sawteeth (2 gyrotrons, $P_{\text {rf }} / P_{\text {beams }} \approx 1 \mathrm{MW} / 6 \mathrm{MW}$, fixed $B_{\mathrm{T}}$ and $R_{\text {surf }}$ ). (a) $\beta_{\mathrm{N}}$ and ECH power, (b) $n=2$ (solid) and $n=1$ (dashed) integrated Mirnov amplitudes. 


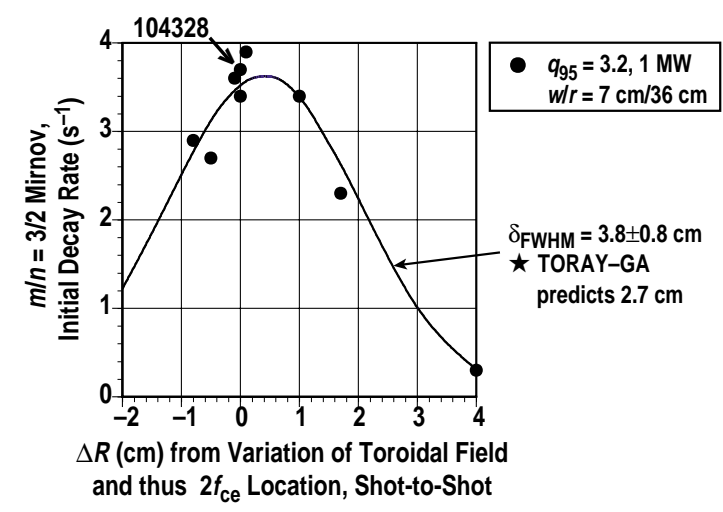

Fig. 6. Initial decay rate of $n=2$ Mirnov amplitude upon application of ECCD versus shift in $2 f_{\text {ce }}$ location (and, thus, relative displacement of island and peak current drive).

\section{REAL-TIME CONTROL OF OPTIMUM POSITION}

To allow for shot-to-shot and time-to-time variation in the optimum position, particularly due to on-axis safety factor $q(0)$ variation with periodic sawteeth affecting the $q=3 / 2$ location, DIII-D has developed real-time control. For fixed $B_{\mathrm{T}}$ and rf launch angles, the plasma control system (PCS) makes small rigid horizontal shifts of the plasma cross-section (and, thus the island) across the peak ECCD. Or, for fixed plasma position $R_{\text {surf }}$, the PCS makes small changes in toroidal field $B_{\mathrm{T}}$ (and thus, the $2 f_{\mathrm{ce}}$ and peak $j_{\mathrm{ec}}$ location) with respect to the island. A "search and suppress" logic looks at the real-time $n=2$ Mirnov signal so as to determine which way and by how much to move the plasma or the $2 f_{\text {ce }}$ resonance to optimize the ECCD suppression.

For fixed $B_{\mathrm{T}}$, the PCS does a $\Delta R$ "blind search" when the Mirnov mode amplitude exceeds a threshold, typically $20 \%$ of the level of the even $n d B_{\theta} / d t$ before the ECCD is applied. The plasma major radius (and island) is rigidly stepped by $\Delta R=1 \mathrm{~cm}$. A dwell of $100 \mathrm{~ms}$ allows for checking if the mode amplitude decreases or not. If yes, but above a threshold, a further step and dwell is made. If no, the next step is in the other direction. Once the mode is suppressed, the PCS freezes $R_{\text {surf }}$ until such time that the mode should reappear. An example of a rigid shift of about $\Delta R=2 \mathrm{~cm}$ is shown in Fig. 7. The $q=3 / 2$ surface follows $R_{\text {surf }}$ (although perhaps with a delay as these times are $\pm 50 \mathrm{~ms}$ around a PCS step command of $2 \mathrm{~cm}$ ); the $2 f_{\text {ce }}$ location has a negligible change. An example of PCS real-time control of $R_{\text {surf }}$ is shown in the "phase plot" of BDOTEVAMPL vs. $R_{\text {surf }}$ in Fig. 8. The discharges are $q_{95}=3.6$ such that the sawteeth become weakly coupled just before the crashes $\left(f_{32} \approx 2 f_{11}\right)$ and three gyrotrons are used for $1.5 \mathrm{MW}$ injected $\left(j_{\mathrm{ec}} / j_{\mathrm{bs}} \approx 2.2\right)$. Complete NTM suppression is achieved (at fixed "optimum" $B_{\mathrm{T}}$ and $\left.R_{\text {surf }}\right)$ in \#106642. Given a demonstration of a condition for complete suppression, the PCS in the example of \#106654 is deliberately started with $\Delta R \approx-2 \mathrm{~cm}$ from "optimum" 


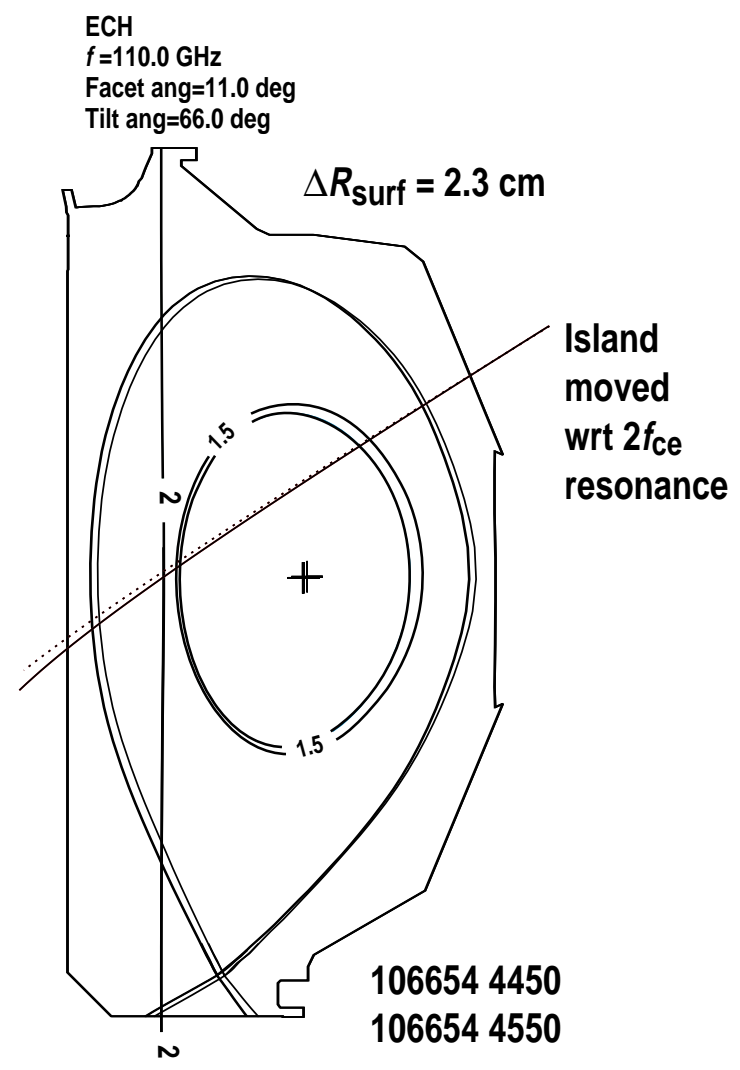

Fig. 7. Rigid shift in major radius by about $2 \mathrm{~cm}$ moves $q=3 / 2$ with negligible change in $2 f_{\text {ce }}$ (and thus, $j_{\text {ec }}$ ) location.

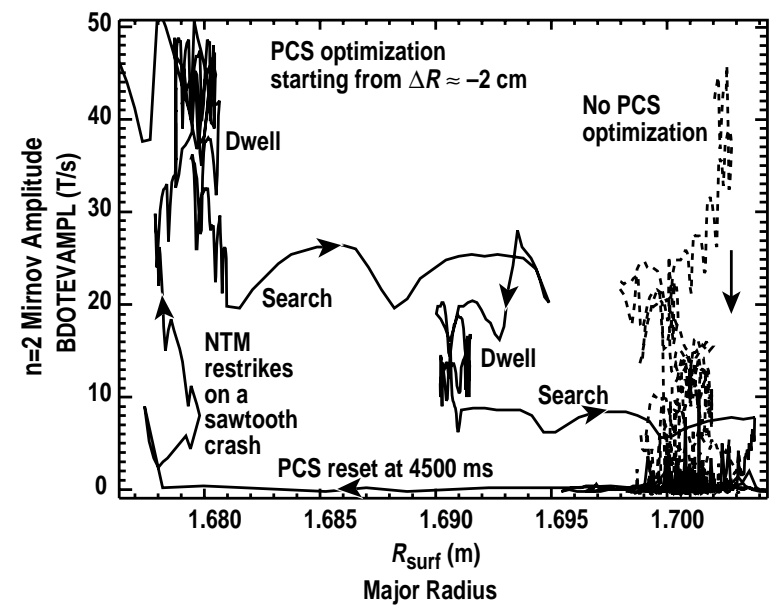

Fig. 8. Trajectory of Mirnov amplitude versus plasma major radius with and without PCS real-time control of optimum rigid plasma position ( $R_{\text {surf }}$ ) for ECCD suppression of an $m / n=3 / 2$ NTM (ECCD with 3 gyrotrons, $1.5 \mathrm{MW}$, on from 3000 to $4800 \mathrm{~ms}, B_{\mathrm{T}}=-1.54 \mathrm{~T}$ flattop, $q_{95}=3.6$ coupled sawtooth case). 
during ECCD and searches and dwells etc. until the optimum $R_{\text {surf }}$ is adjusted and complete suppression obtained. Sawteeth continue but the 3/2 NTM does not restrike with ECCD until at a preset time of $4500 \mathrm{~ms}$ the PCS resets $R_{\text {surf }}$ to the starting point and a sawtooth crash induces the mode with ECCD on but now off-set. This is the difference shown in Fig. 7.

For fixed $R_{\text {surf }}$, the PCS can do a $\Delta B_{\mathrm{T}}$ "blind search". Here the step size is $\Delta B_{\mathrm{T}}=0.01 \mathrm{~T}$ equivalent to $\Delta R=0.9 \mathrm{~cm}\left(\Delta B_{\mathrm{T}} / B_{\mathrm{T}} \approx 0.6 \%\right)$ with the same dwell time of $100 \mathrm{~ms}$. The logic is the same as for $\Delta R$ search at fixed $B_{\mathrm{T}}$. An example of a shift in the $2 f_{\text {ce }}$ resonance of about $2 \mathrm{~cm}$ is shown in Fig. 9. The $q=3 / 2$ location is essentially unchanged. An example of PCS real-time control of $B_{\mathrm{T}}$ is shown in the "phase space plot" of BDOTEVAMPL vs. $B_{\mathrm{T}}$ in Fig. 10. The PCS in the example of \#107390 is deliberately started with $\Delta B_{\mathrm{T}} \approx 0.02 \mathrm{~T}$ or $\Delta R \approx 1.8 \mathrm{~cm}$ from optimum and searches and dwells until the optimum $B_{\mathrm{T}}$ is adjusted and complete suppression obtained.

\section{E. ECCD REQUIREMENTS FOR COMPLETE NTM SUPPRESSION WITH SAWTEETH}

Unlike ASDEX Upgrade or JT-60U, the DIII-D experiments are done with periodic $q=1$ sawteeth present. Initial experiments were done at $q_{95}=4.3, I_{\mathrm{p}}=1.0 \mathrm{MA}$, $B_{T} \approx-1.64 \mathrm{~T}$ so that $q=3 / 2$ was at a smaller normalized minor radius $(\rho \approx 0.53)$ to have higher $T_{\mathrm{e}}$ and more current drive. However with two gyrotrons complete $3 / 2$ NTM suppression could not be achieved. It was recognized that there was no differential rotation between the $q=1$ sawteeth precursors and the $m / n=3 / 2$ NTM, i.e., $2 f_{11} \approx f_{32}$. This suggests nonlinear coupling of the $m / n=3 / 2$ mode to the unstable $m / n=1 / 1$ (and $2 / 2$ ) mode must also be overcome to stabilize the $m / n=3 / 2$ NTM. Discharge adjustment to lower $q_{95}=3.2$, larger minor radius location of $q=3 / 2(\rho \approx 0.63)$ at $I_{\mathrm{p}}=1.2 \mathrm{MA}$, $B_{T} \approx-1.52 \mathrm{~T}$ moved $q=3 / 2$ far enough from the axis so the coupling was broken, i.e., $2 f_{11} \neq f_{32}$. The differential rotation factor $\left(\Delta \omega \tau_{\text {tear }}\right)^{-1 / 2} \approx(250)^{-1 / 2} \approx 0.06<<1$ is sufficient to substantially decouple the surfaces. ${ }^{21}$ [Here $\Delta \omega \approx 2 \pi\left(f_{32}-2 f_{11}\right)$ and $\tau_{\text {tear }}$ is the tearing reconnection time at $q=3 / 2$.] In this case, two gyrotrons were just marginally enough to get complete suppression. An intermediate case $q_{95}=3.6$ at $I_{\mathrm{p}}=$ 1.1 MA and $-1.54 \mathrm{~T}$ was also run with weakly coupled sawteeth, i.e., only $\Delta \omega \approx 0$ near the peak sawteeth precursor amplitude. A summary of the $j_{\mathrm{ec}} / j_{\mathrm{bs}}$ vs $q_{95}$ with best alignment in each case is given in Fig. 11. Typically, a well-aligned level of $j_{\mathrm{ec}} / j_{\mathrm{bs}} \approx 2$ is necessary to get complete $3 / 2$ NTM suppression. 


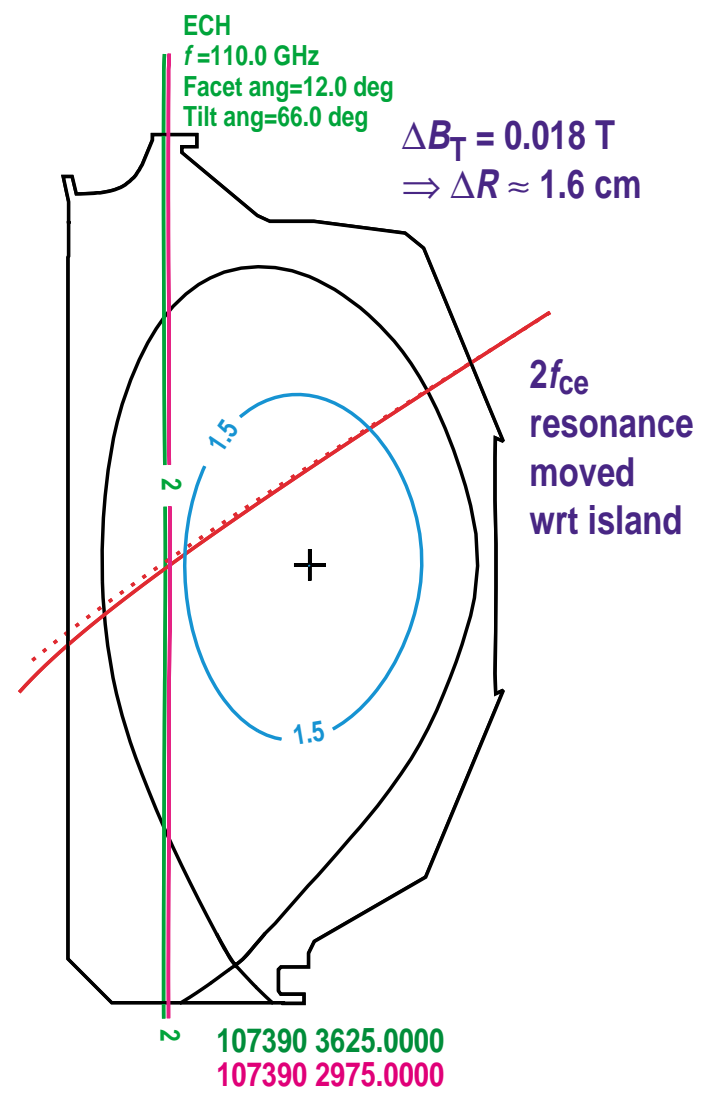

Fig. 9. Shift in $2 f_{\mathrm{ce}}$ (and thus, $j_{\mathrm{ec}}$ ) location by about $2 \mathrm{~cm}$ by $\Delta B_{\mathrm{T}} \approx$ $0.018 \mathrm{~T}(1.1 \%)$ with negligible change in $q=3 / 2$ location.

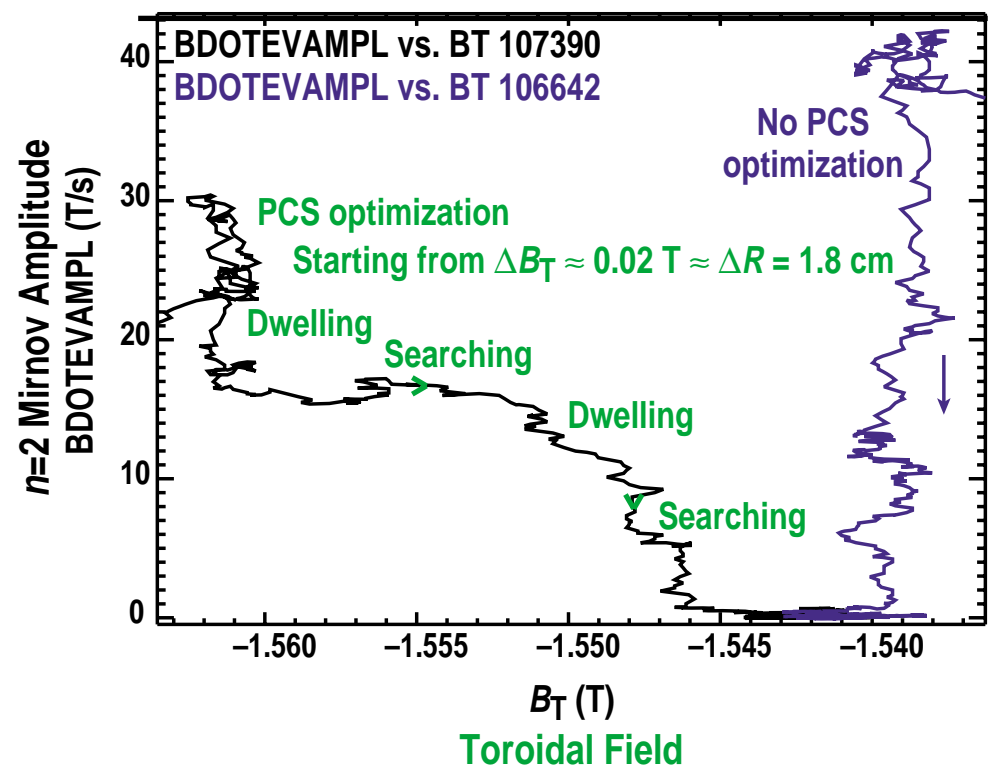

Fig. 10. Trajectory of Mirnov amplitude versus toroidal field with and without PCS real-time control of $2 f$ ce resonance location for ECCD suppression of an $m / n=3 / 2$ NTM (ECCD with 3 gyrotrons, $1.5 \mathrm{MW}$, on from 3000 to $4000 \mathrm{ms,}$ $R_{\text {surf }}=1.700 \mathrm{~m}, q_{95}=3.6$ coupled sawtooth case). 


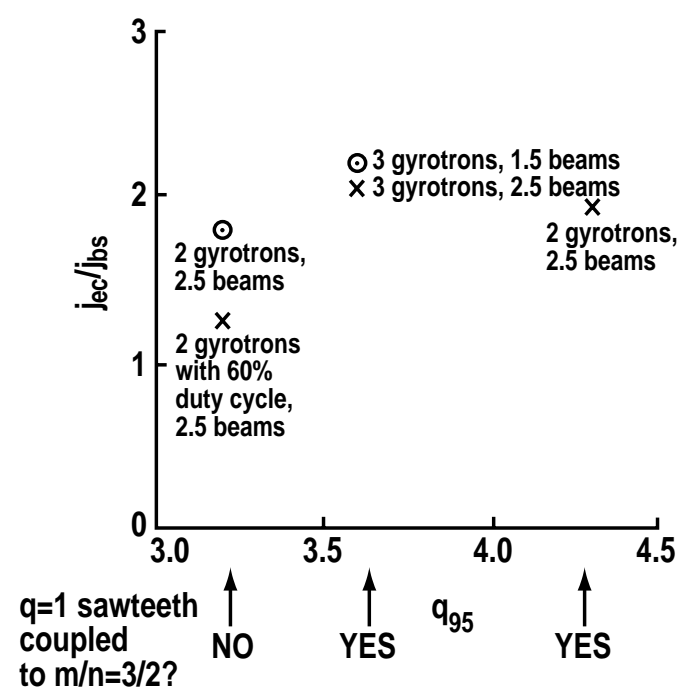

Fig. 11. Ratio of $j_{\text {ec }}$ from TORAY-GA to $j_{\text {bs }}$ from ONETWO for best alignment of ECCD in each case to $m / n=3 / 2$ NTM vs. $q_{95}$. Open circles are complete NTM suppression, $\mathrm{X}$ are partial suppression. 


\section{NON-RESONANT HELICAL FIELD INHIBITION OF AN NTM}

\section{A. MODIFIED RUTHERFORD EQUATION WITH NON-RESONANT FIELD}

The model pressure profiles shown in Fig. 1(b) assume complete flattening inside the island. This maximizes the helically perturbed bootstrap current. However, other effects can make the pressure flattening less than complete, reducing the destabilizing term in Eq. (1a). The usual effect appears as the "transport threshold" island size $w_{\mathrm{d}}$ which for $w_{\mathrm{d}}^{2} \gg w^{2}$ would "wash out" the perturbed bootstrap current.1,11 Increasing $w_{\mathrm{d}} \approx\left(L_{\mathrm{s}} / k_{\theta}\right)^{1 / 2}\left(\chi_{\perp} / \chi_{\|}\right)^{1 / 4}$ would ordinarily take a very large increase in perpendicular transport $\chi_{\perp}$ over the parallel transport $\chi_{\|}$and is both difficult to achieve and undesirable. (Here $L_{\mathrm{s}}=q R / s$ is where $\mathrm{s}$ is the magnetic shear and $k_{\theta}=m / r$.)

A helical field of a different helicity ( $\operatorname{cuch}$ as $m / n=3 / 2$ ) from a different NTM was found to suppress a pre-existing NTM (such as $m / n=4 / 3$ ) in ASDEX Upgrade if the additional NTM was large enough in amplitude and the rational surfaces close enough. ${ }^{12}$ Theoretical explanation of these experiments led to proposing a theory of NTM suppression by "large" externally applied helical modes. ${ }^{13}$ Preferably these applied fields from a helical coil should be non-resonant with any $q=m / n$ surface in the plasma so as to drive no reconnection. It should be noted that preliminary experiments with resonant $m / n=1 / 1$ and $2 / 2$ fields are under invesitgation at JET. ${ }^{22}$ The simplest way to view the physics of NTM suppression by another helicity is: (1) the applied field bends field lines at the NTM island chain, (2) the bending puts a small fraction $|\tilde{b}|$ of the very large parallel heat transport $\chi_{\|}$in the "perpendicular" direction to add to $\chi_{\perp}$. (Here $|\tilde{\mathrm{b}}| \equiv\left|\tilde{B}_{\text {rmn }}\right| / B_{\text {To }}$ is the ratio of the applied helical radial field at the NTM surface to the equilibrium toroidal field $B_{\mathrm{To}}$ ). This "enhanced" $\chi_{\perp}$ interferes with the fundamental harmonic of the NTM in the same way as the $w_{\mathrm{d}}$ transport threshold in Eq. (1a). The effective $w_{\mathrm{d}}$ is 13

$$
w_{\mathrm{d}}=w_{\mathrm{do}}\left(1+\frac{|\tilde{b}|^{2}}{4 \chi_{\perp} / \chi_{\|}}\right)^{1 / 4},
$$

where $w_{\mathrm{do}}=\left(L_{\mathrm{s}} / k_{\theta}\right)^{1 / 2}\left(\chi_{\perp} / \chi_{\|}\right)^{1 / 4}$ as before. For $\chi_{\perp} / \chi_{\|}=2 \times 10^{-8}$ and $|\tilde{b}|=2 \times 10^{-3}$, $w_{\mathrm{d}}$ is increased by a factor of 3 . This is enough weakening of the helically perturbed bootstrap destabilization to completely suppress a typical NTM as shown in the evaluation of the modified Rutherford equation in Fig. 12. 
For less $|\tilde{b}|$ there would be partial suppression of a previously saturated NTM and complete inhibition before onset of not too large a seed island. This hysteresis is explained in Fig. 13 by plotting the perturbed bootstrap current only vs. island size at different applied helical field. For large saturated islands, $w \approx 7 \mathrm{~cm}$ for example, $|\tilde{b}|$ has a weak effect as $\mathrm{w}^{2}>>\mathrm{w}_{\mathrm{d}}^{2}$ even for large $|\tilde{b}|=2 \times 10^{-3}$. However, for small seed islands, $w \lesssim 2 \mathrm{~cm}$ typically, ${ }^{1}$ the destabilizing term is substantially weakened.

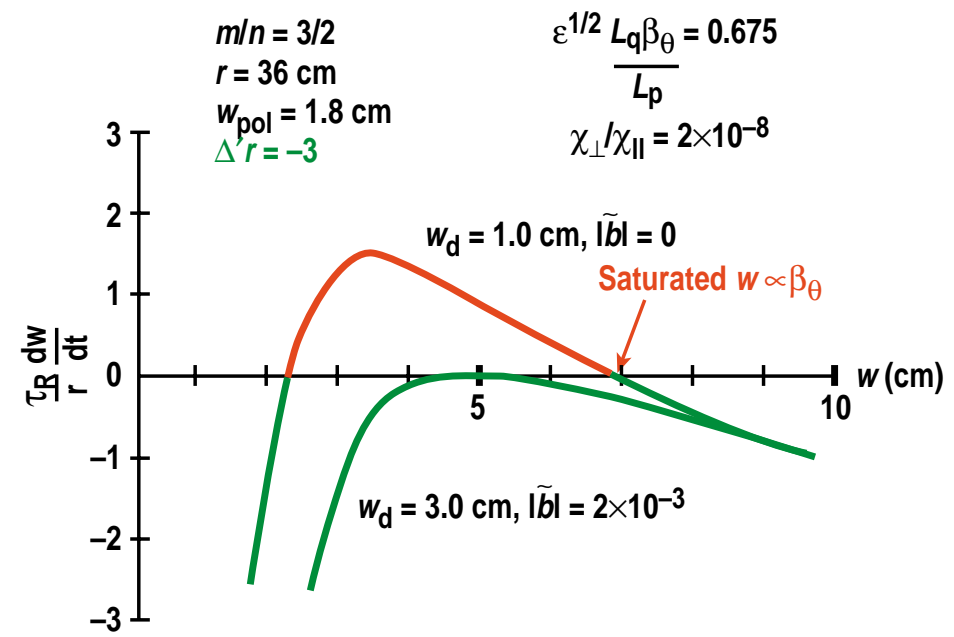

Fig. 12. Modified Rutherford equation evaluated with and without $(|\tilde{b}|=0)$ external helical field applied.

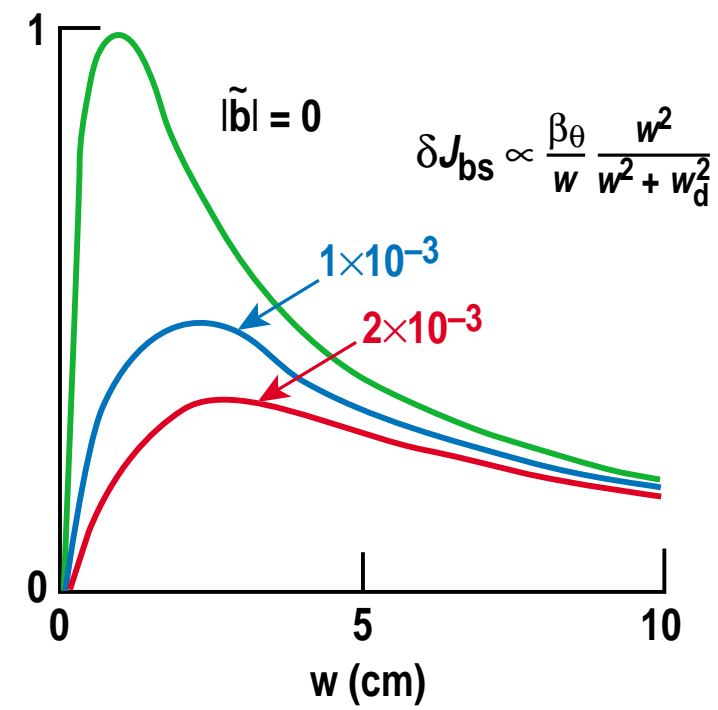

Fig. 13. Perturbed bootstrap current vs. island size $w$ for different levels of external helical field $|\tilde{b}|$ applied. Here $w_{\mathrm{d}}=1 \mathrm{~cm}$ for $|\tilde{b}|=0$. 


\section{B. NON-RESONANT HELICAL FIELD FROM THE "C-COIL"}

The "C-Coil" on DIII-D is used to apply toroidally asymmetric magnetic fields. ${ }^{23,24}$ The six section coil, shown in Fig. 14(a), is used in this study to apply an $n=3$ magnetic field. Both $n=1$ resonant error field correction and $n=3$ fields can be applied simultaneously depending on the relative coil currents. Each section is four turns with a maximum current allowed of $5000 \mathrm{~A}$. The $n=3$ helical field is predominantly nonresonant, as confirmed by Fourier analysis on the highlighted surface in Fig. 14(a) which is about at normalized minor radius $\rho=0.6$. For safety factor on axis $q_{0} \approx 1$ and $q$ monotonically increasing with radius, i.e., $q \geq q_{0} \approx 1$ everywhere, the dominant helicities from the $\mathrm{C}-$ Coil are $m / n=1 / 3$ and $2 / 3$ and non-resonant.

The $n=3$ field from the C-Coil is similar to a "ripple" field from toroidal field coils but at much lower $n$. [There is $n=24$ ripple of $\pm 0.55 \%$ of $B_{\mathrm{T}}$ at the outboard midplane boundary of DIII-D $(R=2.34 \mathrm{~m})$ which falls off rapidly as $\left(R / R_{\mathrm{TF}}\right)^{24}$ to only $1.5 \times 10^{-4} \%$ at $R=2.06 \mathrm{~m}, \rho \approx 0.6$ where $R_{\mathrm{TF}}=2.90 \mathrm{~m}$ is the outboard midplane location of the toroidal field coil. ${ }^{25}$ ] The $n=3$ field radial fall off is shown in Fig. 14(c) to be quite gradual and the field is significant across the plasma volume.

\section{APPLICATION OF $n=3$ FIELD AFTER A SATURATED $m / n=3 / 2$ NTM}

As in the ECCD work, an $m / n=3 / 2$ NTM was excited on a sawtooth crash and allowed to evolve to a large saturated island at constant neutral beam power. The plasma shape was as in Fig. 3, with $q_{95}=3.7$ and "weakly" coupled sawteeth as in Figs. 7 and 10. However, as tuning to the $2 f_{\text {ce }}$ resonance was not necessary, plasmas were run at lower toroidal field and plasma current so as to allow $|\tilde{b}|$ to be larger. $\left(I_{\mathrm{p}}=750 \mathrm{kA}, B_{\mathrm{T}}=\right.$ $-1.1 \mathrm{~T}, \bar{n}=5.7 \times 10^{13} \mathrm{~cm}^{-3}, \beta_{\mathrm{N}}=2.2, \beta_{\theta}=0.95$, with $T_{\mathrm{e}}=0.85 \mathrm{keV}$ and $T_{\mathrm{i}}=0.89 \mathrm{keV}$ at the $q=3 / 2$ surface.) The island size $w$ estimated from Mirnov analysis is about $7 \mathrm{~cm}$ full width or $w / r \approx 20 \%$. The critical island $w_{\mathrm{d}}$ for incomplete pressure flattening is about $0.8 \mathrm{~cm}$ with $\chi_{\perp} \approx 1.7 \mathrm{~m}^{2} / \mathrm{s}$ at $\rho=0.5$ from ONETWO transport analysis, $\chi_{\|} \approx 7.7 \times 10^{7} \mathrm{~m}^{2} / \mathrm{s}$ from ion collisional transport (mean freepath $\lambda_{\mathrm{mfp}}=\mathrm{v}_{\mathrm{i}} / v_{\mathrm{ii}} \approx 260 \mathrm{~m}$ less than parallel connection length $\lambda_{\|}=4 \pi L_{\mathrm{s}} / k_{\theta} w_{\mathrm{d}} \approx 620 \mathrm{~m}$ where $L_{\mathrm{s}}=q R / \mathrm{s}=4.2 \mathrm{~m}$ for shear $s=0.6$ and $k_{\theta}=m / r=9.4 \mathrm{~m}^{-1}$ for $m=3$ and $\left.r=0.32 \mathrm{~m}\right) .{ }^{1}$ Note that the banana ions are in the trapped regime as $\mathrm{v}_{\mathrm{ii}} / \varepsilon \omega_{\mathrm{bi}} \approx 0.1<<1$ for $\omega_{\mathrm{bi}}=\varepsilon^{1 / 2} \mathrm{v}_{\mathrm{i}} / q R$ the ion bounce frequency. 26

For evaluation of the effective non-resonant $n=3$ helical field from all $m$ components, both left and right handed, the Fourier components of Fig. 14(b) are added in quadrature as $B_{\mathrm{r} 3 \mathrm{eff}}$ as each separate component should theoretically act cumulatively in 

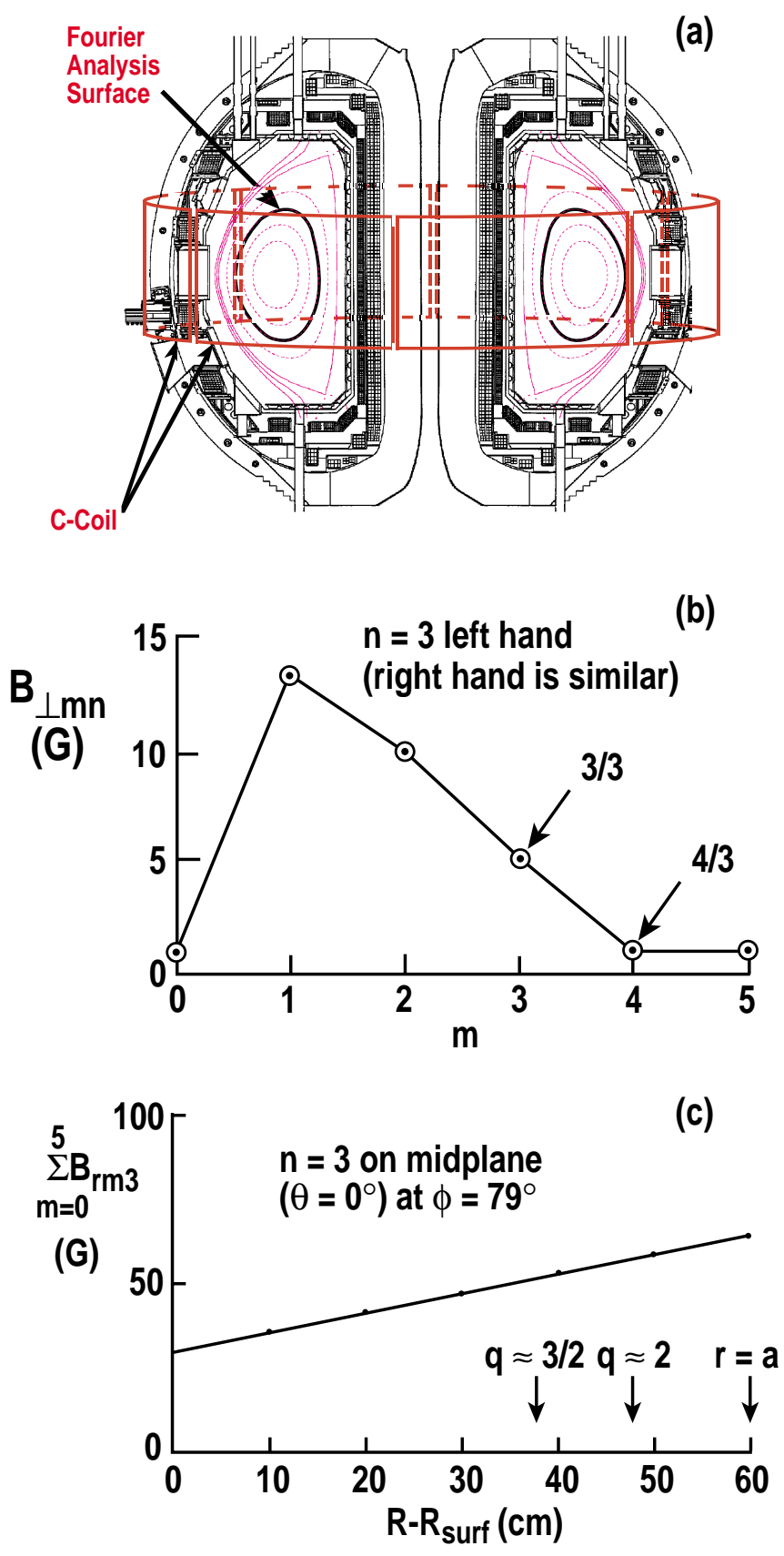

Fig. 14. (a) The six section C-Coil. Also shown is surface for Fourier analysis; (b) Fourier analysis at $\rho \approx 0.6$ of perpendicular ("radial") $n=3$ magnetic field at maximum C-Coil current of 5000 A; (c) Gradual fall off of total $n=3$ radial field $\left(\sum B_{\mathrm{rm} 3}\right)$ on midplane location. $R_{\text {surf }}$ is major radius of plasma.

Eq. (2). ${ }^{27}$ Finally, we also here introduce the effective $m / n=2 / 1$ resonant error field $B_{\text {pen21 }}$ which can induce rotation drag, locking or reconnection at $q=2$ and which the $\mathrm{C}-$ Coil is routinely used to minimize. ${ }^{24}$ 
The result of switching on the $n=3$ field to $|\tilde{b}| \equiv B_{\mathrm{r} 3 \mathrm{eff}} / B_{\mathrm{T} 0} \approx 1.6 \times 10^{-3}$ in $500 \mathrm{~ms}$, about three confinement times, is shown in Fig. 15. The three $\mathrm{C}-\mathrm{Coil}$ pairs are all at maximum currents of $-5000,5000$, and -5000 A respectively at $\phi=79,139$ and 199 degrees. The principal effect is a reduction in plasma rotation [Fig. 15(c)] to be discussed in the next section. While there is a transient dip in $n=2$ Mirnov amplitude [at $3200 \mathrm{~ms}$, Fig. 15(d)] this is not maintained and $\beta_{N}$ (and $\tau_{E}$ ) drop by about $20 \%$. The reduction in $\beta_{\mathrm{N}}$ is due to a similar decrease in density (no filling gas is input) with central $T_{\mathrm{i}}$ (and $T_{\mathrm{e}}$ ) about unchanged and neutron production (deuterium plasma) also about unchanged. As indicated in Fig. 13, $|\tilde{b}| \approx 1.6 \times 10^{-3}$ appears to be insufficient to make a significant effect on a large $(w \approx 7 \mathrm{~cm})$ island. Additional experiments used lower $B_{\mathrm{T}}(-1.0 \mathrm{~T})$ and $q_{95}$ (3.2) which both somewhat increase $|\tilde{b}| \quad(q=3 / 2$ moves to larger minor radius at lower $q_{95}$ ) and larger transient dips in $n=2$ Mirnov amplitude were obtained but only in long ELM-free periods. Results and modeling of these other discharges will be reported elsewhere. The plasma parameters of Fig. 15 were repeated in the inhibition, i.e., early, low $\beta_{\theta} n=3$ field application before the $n=2$ NTM, and are discussed in the section after next.

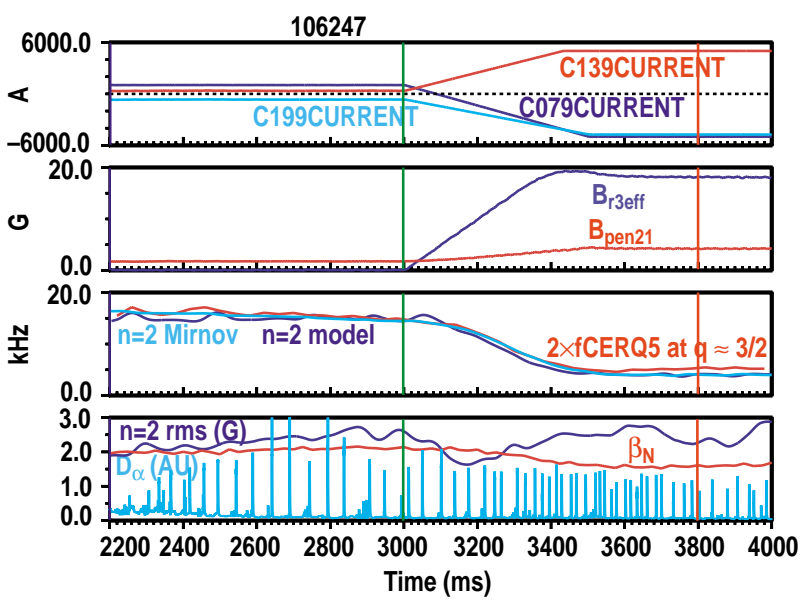

Fig. 15. (a) C-Coil currents switched to make $n=3$ field starting at $3000 \mathrm{~ms}$; (b) effective $n=3$ field $B_{\mathrm{r} 3 \mathrm{eff}} \approx 18 \mathrm{G}$ for $|\tilde{b}| \approx 1.6 \times 10^{-3}$ at $\left|B_{\mathrm{T}}\right|=1.1 \mathrm{~T}$; (c) rotation drops as shown by $n=2$ Mirnov frequency (solid line) and twice ion toroidal rotation frequency at chord \#5 which is closest to $q=3 / 2$ (dotted line) with $n=2$ ripple damping rotation model (dashed line) also shown; (d) $n=2$ integrated Mirnov amplitude, $\beta_{\mathrm{N}}$ (dotted line) and $\mathrm{D}_{\alpha}$ light.

\section{ROTATION REDUCTION WITH $n=3$ FIELD}

The rotation reduction as the $n=3$ field is turned on is prompt and eventually reduces the global momentum confinement time $\tau_{M}$ by $83 \%$. The reduction is across the entire profile as shown in Fig. 16 as measured by charge exchange recombination (CER) 


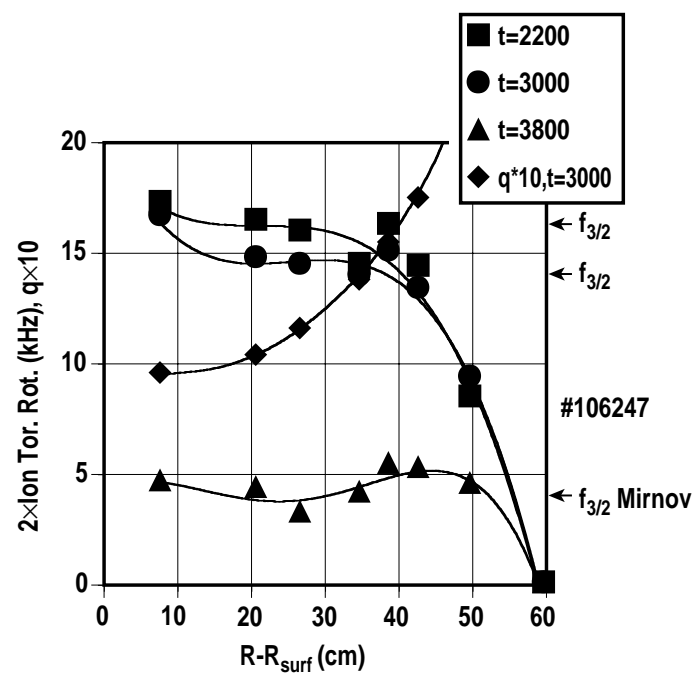

Fig. 16. Profiles of twice (for $n=2$ comparison) ion toroidal rotation frequency with $m / n=3 / 2$ NTM at times (2200 and 3000) before $n=3$ field and also at 3800 with $n=3$ field. $R_{\text {surf }}=170 \mathrm{~cm}$, the $q$ profile from EFIT is included and $f_{3 / 2}$ is the Mirnov frequency at each time (2200 highest, 3800 lowest).

analysis of CVI ion toroidal rotation. The ion toroidal rotation is in good agreement with the $n=2$ Mirnov frequency before and after the $n=3$ field is turned on.

The decrease in rotation is not compatible with an induction motor model involving the drag of resonant fields and slipping. ${ }^{29,30}$ In that model, a resonant $m, n$ helical field acts on a slipping, rotating plasma at the rational surface $q=m / n$ to induce an $m, n$ helical current sheet which opposes reconnection but whose small phase shift due to finite reconnection/tearing time $\tau_{\text {rec }}$ leaves a net $\vec{J} \times\left.\vec{B}\right|_{\mathrm{m}, \mathrm{n}}$ drag. In an induction motor model for natural rotation $f_{\mathrm{O}}$ and confinement time $\tau_{\mathrm{Mo}}$

$$
\frac{d f}{d t}=\frac{f_{\mathrm{O}}-f}{\tau_{\mathrm{Mo}}}-\frac{C_{\mathrm{ind}} B_{\mathrm{rmn}}^{2}}{\mathrm{f}},
$$

for $m / n=2 / 1$ for example, with

$$
\frac{f}{f_{\mathrm{o}}} \approx \frac{1}{2}\left(1-\frac{\tau_{\mathrm{Mo}} \dot{f}}{f_{\mathrm{o}}}\right)+\frac{1}{2}\left[\left(1-\frac{\tau_{\mathrm{Mo}} \dot{f}}{f_{\mathrm{o}}}\right)^{2}-\frac{4 \tau_{\mathrm{Mo}} \mathrm{C}_{\mathrm{ind}} B_{\mathrm{r} 21}^{2}}{f_{\mathrm{o}}^{2}}\right]^{1 / 2},
$$

and locking, i.e. a decrease in $f$ of $50 \%$, occurs for $B_{\mathrm{r} 21}^{2} \approx f_{\mathrm{o}}^{2} / 4 C_{\text {ind }} \tau_{\mathrm{Mo}}$. This model fits neither the larger rotation decrease nor the time dependence.

The switch to maximum $n=3 \mathrm{C}$-Coil currents in Fig. 15 turned off the $n=1$ error field correction causing $B_{\text {pen21 }}$ to increase to the intrinsic uncorrected error field level. 
For co-injected beam driven rotation this should not be a significant enough $2 / 1$ field to damp the rotation. However, checks were made. In another discharge, the $n=1 \mathrm{C}-\mathrm{Coil}$ correcting currents were maintained and the $n=3$ currents increased to about $80 \%$ of the level in Fig. 15. A large rotation reduction was still observed. In yet another discharge, no $n=3$ field was applied but the $\mathrm{n}=1$ correction slowly turned off and then the $n=1$ field increased as in magnetic braking; a substantially higher $B_{\text {pen21 }}$ than in \#106247 was needed to reduce rotation by $50 \%$ to the locking level of Eq. (3b) consistent with a resonant error field. Thus, the tests indicate that the rotation damping is not due to an applied resonant field.

What does fit the rotation reduction is ripple damping of beam-induced toroidal plasma rotation. The closest related low $\mathrm{n}$ ripple experiment is the use of nine toroidal field (TF) coils in ISX-B (the standard was 18) which observed a reduction of central toroidal rotation velocity by a factor of six and momentum confinement time a factor of seven. ${ }^{31}$ The $n=9$ ripple, peak to average field, was $2 \%$ to $10 \%$ from the plasma axis to the outboard midplane boundary. The $n=3$ ripple for DIII-D \#106247 is similarly about $0.3 \%$ to $0.9 \%$. Detailed modeling of the $n=3$ ripple drag is beyond the scope of this paper and will be reported on elsewhere. However, modeling of ripple drag 32 related to "transient time magnetic pumping," has a form

$$
\frac{d f}{d t}=\frac{f_{\mathrm{o}}-f}{\tau_{\mathrm{Mo}}}-C_{\text {rip }} f B_{\mathrm{r} 3 \mathrm{eff}}^{2},
$$

for the $n=3$ effective field for example, with

$$
\frac{f}{f_{\mathrm{o}}} \approx \frac{\left(1-\tau_{\mathrm{Mo}} \dot{f} / f_{\mathrm{o}}\right)}{1+\tau_{\mathrm{Mo}} C_{\mathrm{rip}} B_{\mathrm{r} 3 \mathrm{eff}}^{2}} .
$$

The fit to the model in Fig. 15(c) has $f_{\mathrm{o}}=15 \mathrm{kHz}, \tau_{\mathrm{Mo}}=0.19 \mathrm{~s}$ and $C_{\text {rip }}=0.029 \mathrm{~s}^{-1} \mathrm{G}^{-2}$. Note that the form of Eq. (4b) has no critical condition for locking and that the rotation decreases continuously as $B_{\mathrm{r} 3 \mathrm{eff}}$ is increased.

In consideration of drag from $n=3$ transit time magnetic pumping in the frame moving with the plasma at toroidal velocity $\mathrm{V}_{\phi}\left(\omega=k_{\phi} \mathrm{V}_{\phi}\right.$ where $k_{\phi}=n / R$ and $\left.n=3\right)$, the plasma at the $q=3 / 2$ outboard midplane location $\left\{R_{\text {out }}=2.07 \mathrm{~m},\left|B_{\mathrm{T}}\right|=0.9 \mathrm{~T}, n=3\right.$ ripple field $B_{\mathrm{r}}^{(1)}=50 \mathrm{G}$ [from Fig 14(c)] at maximum $\mathrm{C}-$ Coil current ] is in the regime $\mathrm{V}_{\mathrm{i}} \gg \mathrm{V}_{\phi} \gg \mathrm{V}_{\mathrm{D} \phi}$. Here $\mathrm{V}_{\mathrm{i}}=\left(2 k_{\mathrm{B}} T_{\mathrm{i}} / m_{\mathrm{i}}\right)^{1 / 2} \approx 300 \mathrm{~km} / \mathrm{s}$ is the ion thermal speed, $\mathrm{V}_{\phi} \approx 100 \mathrm{~km} / \mathrm{s}$ is the ion toroidal flow (before turning on the $n=3$ field) and $\mathrm{V}_{\mathrm{D} \phi} \approx 20 \mathrm{~km} / \mathrm{s}$ is the ion toroidal drift speed $\left|k_{\mathrm{B}} T_{\mathrm{i}} / e B_{\theta} L_{\mathrm{Ti}}\right|$. The $n=3$ ripple field 
exerts drag on the plasma rotation which, using Eq. (57) of Ref. 32 can be put into the form

$$
\left.\frac{d f}{d t}\right|_{\mathrm{TTMP}}=-\sqrt{\frac{\pi}{4}} \mathrm{~V}_{\mathrm{i}} \frac{n}{R_{\mathrm{out}}}\left(\frac{n+1}{n}\right)^{2}\left[\frac{R_{\mathrm{out}}}{(2 n+2)\left(R_{\mathrm{out}}-R_{\mathrm{in}}\right)}\right]\left(\frac{B_{\mathrm{r}}^{(1)}}{B_{\mathrm{T}}}\right)^{2} f,
$$

evaluated at the outboard midplane $q=3 / 2$ radius $R_{\text {out }}(2.07 \mathrm{~m})$ where the [ ] averages the combined major radial variations in $k_{\phi}, B_{\mathrm{r}}^{(1)}$, and $B_{\mathrm{T}}$ from $R_{\text {out }}$ to the inboard $q=3 / 2$ radius $R_{\text {in }}(1.43 \mathrm{~m})$. At full $\mathrm{C}-$ Coil current $d f /\left.d t\right|_{\mathrm{TTMP}}$ from Eq. (5) $\approx-8.6 \mathrm{~s}^{-1} * f$ compared to the empirical fit drag term from Eq. (4a) of $-C_{\text {rip }} B_{\mathrm{r} 3 \mathrm{eff}}^{2} f \approx-9.4 \mathrm{~s}^{-1} * f$.

\section{E. APPLICATION OF $n=3$ FIELD BEFORE THE $m / n=3 / 2$ NTM ONSET}

Given the difficulty for the $n=3$ field in affecting a large saturated NTM $\left(w^{2}>>w_{\mathrm{d}}^{2}\right)$, as indicated in Fig. 13, the same parameter plasmas as in \#106247 of Fig. 15 were run but with the $n=3$ field turned on in the low $\beta_{\theta}$ phase before the neutral beams increase beta so that the discharges could start in the regime $w^{2}<<w_{\mathrm{d}}^{2}$ with large enough $n=3$ field. Three discharges are shown in Fig. 17, \#106339 with the maximum $n=3$ field (as in \#106247), \#106336 with 0.5 times the maximum $n=3$ field and \#106334 with no $n=3$ field. The maximum $n=3$ field inhibits the onset of the $m / n=3 / 2$ NTM until late in the discharge when the $n=3$ field is ramped off and the NTM appears. At half the maximum $n=3$ level, the NTM is not inhibited and appears early in the discharge. In both $n=3$ cases, the plasma rotation is reduced as before. The consistent critical level for inhibition is $|\tilde{b}| \equiv B_{\mathrm{r} 3 \mathrm{eff}} / B_{\mathrm{T} 0} \approx 1.2 \times 10^{-3}$. Note that based both on $n=3$ Mirnov analysis and on electron cyclotron emission data, all of these discharges have frequent (every $50 \mathrm{~ms}$ ) sawteeth of about the same amplitude which can act as $m / n=3 / 2$ NTM seeds. Suppression of the $m / n=3 / 2$ NTM does not increase $\beta_{\mathrm{N}}$ which may be due to the offsetting reduction in confinement caused by the reduced rotation in $\mathrm{H}$-mode. 


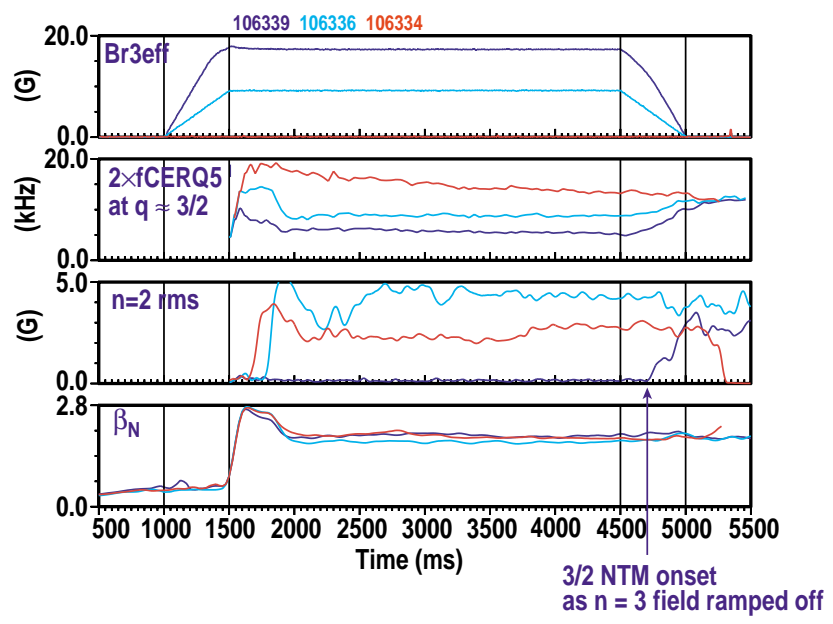

Fig. 17. Different levels of $n=3$ field applied before neutral beams raise beta. Same parameters as in $\# 106247$ of Fig. $15 . B_{\text {r3eff }}$ is effective $n=3$ field, twice toroidal rotation measured by CER at about $q=3 / 2, n=2$ rms Mirnov amplitude and $\beta_{\mathrm{N}}$. 


\section{CONCLUSIONS}

\section{A. ECCD NTM CONTROL}

Large ( $w / r \approx 20 \%$ full width) $m / n=3 / 2$ neoclassical tearing modes in the presence of sawteeth are completely suppressed by precisely located (to $\pm 1 \mathrm{~cm}$ ) off-axis electron cyclotron current drive. A level of peak $j_{\mathrm{ec}}$ of about twice $j_{\mathrm{bs}}$ is required for stabilization. A little higher value may be necessary if there is direct coupling of $q=1$ and $q=3 / 2$ surfaces. The DIII-D plasma control system (PCS) has been developed to do real-time search and suppress position control using either rigid radial plasma shifts or adjustment of the toroidal field and thus second harmonic electron cyclotron resonance location. This represents the first use of active feedback control to position the ECCD. After NTM suppression, with continued ECCD, beta could be substantially raised above the onset level. Future work is to apply ECCD to inhibit the onset of NTMs and will require the PCS to locate the $q=3 / 2$ surface, for example, and position the ECCD in the absence of the NTM, particularly as beta is increased and a Shafranov shift occurs. Future work will also be to suppress the $m / n=2 / 1$ NTM.

\section{B. $n=3$ NON-RESONANT FIELD NTM CONTROL}

A large $|\tilde{b}| \approx 1.2 \times 10^{-3}$ non-resonant field can inhibit the onset of neoclassical tearing modes in sawteething discharges which would otherwise have large $(w / r \approx 20 \%)$ $m / n=3 / 2$ NTMs. The saturated NTMs could not be suppressed at $|\tilde{b}| \leq 1.6 \times 10^{-3}$. This method has the advantages that no precise positioning is needed and that it can act on multiple possible NTMs simultaneously (4/3, 3/2, 2/1, etc.) The disadvantage is that the plasma rotation is strongly damped. Future work is to make use of proposed C-Coil extensions poloidally above and below the existing $\mathrm{C}-\mathrm{Coil}$ to examine if moving the peak $n=3$ ripple off the outboard midplane, for example, can reduce the rotation damping. 


\section{REFERENCES}

${ }^{1}$ R.J. La Haye, R.J. Buttery, S. Günter, G.T.A. Huysmans, M. Maraschek, and H.R. Wilson, Phys. Plasmas 7, 3349 (2000).

${ }^{2}$ S. Günter, A. Gude, M. Maraschek, Q. Yu, Plasma Phys. Control. Fusion 41, 767 (1999).

${ }^{3}$ R.J. La Haye, L.L. Lao, E.J. Strait, T.S. Taylor, Nucl. Fusion 37, 397 (1997).

${ }^{4}$ W.X. Qu and J.D. Callen, University of Wisconsin Report UWPR85-5 (1985).

${ }^{5}$ R. Carrera, R.D. Hazeltine, M. Kotschenreuther, Phys. Fluids 29, 899 (1988).

${ }^{6}$ C.C. Hegna and J.D. Callen, Phys. Plasmas 4, 2940 (1997).

${ }^{7}$ H. Zohm, Phys. Plasmas 4, 3433 (1997).

${ }^{8}$ G. Gantenbein, H. Zohm, G. Giruzzi, S. Günter, F. Leuterer, M. Maraschek, J. Meskat, W. Suttrop, M. Zabiego, Phys. Rev. Lett. 85, 1242 (2000).

${ }^{9}$ A. Isayama, Y. Kamada, S. Ido, K. Hamamatsu, T. Oikawa, T. Suzuki, Y. Neyatani, T. Ozeki, Y. Ikeda, K. Kajiwara, Plasmas Phys. Control. Fusion 42, L37 (2001).

10ITER Physics Basis Editors, Nucl. Fusion 39, 2137 (1999).

${ }^{11}$ R. Fitzpatrick, Phys. Plasmas 2, 825 (1995).

${ }^{12}$ Q. Yu, S. Günter, K. Lackner, A. Gude, M. Maraschek, Nucl. Fusion 40, 2031 (2000).

${ }^{13}$ Q. Yu, S. Günter, K. Lackner, Phys. Rev. Lett. 85, 2949 (2000).

${ }^{14}$ T.C. Luce, Y.R. Lin-Liu, R.W. Harvey, G. Giruzzi, P.A. Politzer, et al., Phys. Rev. Lett. 83, 4550 (1999).

15T.C. Luce, Y.R. Lin-Liu, R.W. Harvey, G. Giruzzi, J.M. Lohr, et al., Plasma Phys. and Control. Fusion 41, B119 (1999).

${ }^{16}$ C.C. Petty, Y.R. Lin-Liu, T.C. Luce, M.A. Makowski, R. Prater, et al., Nucl. Fusion 41, 551 (2001).

${ }^{17}$ K. Matsuda, IEEE Trans. Plasma Sci. P S-17, 6 (1989).

18H.E. St. John, T.S. Taylor, Y.R. Lin-Liu, A.D. Turnbull, Plasma Phys. and Control.

Nucl. Fusion Research 1994, Seville, Spain, Vol. 3 (International Atomic Energy Agency, Vienna, 1995) p. 603.

${ }^{19}$ R.W. Harvey, W.M. Nevins, G.R. Smith, B. Lloyd, M.R. O’Brien, C.D. Warrick, Nucl. Fusion 37, 69 (1997).

20T.A. Gianakon, C.C. Hegna, J.D. Callen, Bull. Am. Phys. Soc. 41, 1371 (1996).

${ }^{21}$ C.C. Hegna, J.D. Callen, R.J. La Haye, Phys. Plasmas 6, 130 (1999).

22T.C. Hender, private communication (2001).

${ }^{23}$ R.J. Buttery, M. De Benedetti, D.A. Gates, Y. Gribov, T.C. Hender, et al., Nucl. Fusion 39, 1827 (1999). 
${ }^{24}$ J.T. Scoville and R.J. La Haye, "Multi-Mode Error Field Correction in the DIII-D Tokamak," to be submitted to Nucl. Fusion, General Atomics Report GA-A23847.

25 J.A. Leuer, "DIII-D TF Coil Ripple: Infinite Conductor Approximation," General Atomics DIII-D Physics Memo 9204 (1992).

26J.W. Connor, R.J. Hastie, Nucl. Fusion 13, 221 (1973).

${ }^{27} \mathrm{Q}$. Yu, private communication (2001).

${ }^{28}$ R.J. La Haye, C.L. Rettig, R.J. Groebner, A.W. Hyatt, and J.T. Scoville, Phys. Plasmas 1, 373 (1994).

${ }^{29}$ R. Fitzpatrick, Phys. Plasmas 5, 3325 (1998).

${ }^{30}$ R.J. La Haye, A.W. Hyatt, and J.T. Scoville, Nucl. Fusion 32, 2119 (1992).

${ }^{31}$ S.D. Scott, J.F. Lyon, J.K. Munro, D.J. Sigmar, S.C. Bates, et al., Nucl. Fusion 25, 359 (1985).

32T.H. Stix, The Theory of Plasma Waves, p. 207 (McGraw-Hill Book Company, New York, 1962). 


\section{ACKNOWLEDGMENTS}

This work was supported by the U.S. Department of Energy under Contract No. DEAC03-99ER54463. The authors would like to thank the DIII-D operations and diagnostic teams which made this work possible. Discussions are appreciated with P.A. Politzer on transit time magnetic pumping damping of rotation and with $\mathrm{Q}$. Yu on the effective field from multiple non-resonant harmonics. 